\title{
Overexpression of tobacco ethylene response factor $N t E R F 3$ gene and its homologues from tobacco and rice induces hypersensitive response-like cell death in tobacco
}

\author{
Takuya Ogata $\cdot$ Yuma Kida $\cdot$ Tomoyuki Arai • \\ Yasuyuki Kishi $\cdot$ Yuki Manago • Masato Murai • \\ Yasuhiko Matsushita
}

Received: 28 September 2011/Accepted: 23 November 2011/Published online: 17 December 2011

(C) The Author(s) 2011. This article is published with open access at Springerlink.com

\begin{abstract}
Among plant-specific transcription factors, ethylene response factors (ERFs) comprise one of the largest families. ERFs are unique to the plant kingdom and are considered to have crucial roles in plant response to various biotic and abiotic environmental stresses. Here, we report on the functional analysis of a transcriptional repressor, NtERF3, with regard to cell death associated with a hypersensitive response (HR), a plant-specific resistance reaction against pathogens. Expression of NtERF3 was upregulated during HR induction by Tobacco mosaic virus (TMV) infection in tobacco plants harboring the resistance $N$ gene to TMV. Transient overexpression of NtERF3 by Agrobacterium-mediated gene delivery induced HR-like cell death in tobacco, associated with the production of reactive oxygen species and ion leakage. Deletion of the ERF-associated amphiphilic repression (EAR) motif from NtERF3 resulted in no induction of cell death, while the deletion had no effect on nuclear localization of the proteins. After virus-mediated gene delivery, similar results also were observed in tobacco without the $N$ gene. In addition to NtERF3, other EAR motif-containing ERFs from tobacco, Nicotiana benthamiana and rice also induced cell death when overproduced in tobacco
\end{abstract}

The nucleotide sequence data reported are available in the DDBJ/ EMBL/GenBank databases under the accession numbers AB573716, AB573717, AB573718 and AB573719.

Electronic supplementary material The online version of this article (doi:10.1007/s10327-011-0355-5) contains supplementary material, which is available to authorized users.

T. Ogata - Y. Kida - T. Arai - Y. Kishi - Y. Manago ·

M. Murai · Y. Matsushita $(\bowtie)$

Gene Research Center, Tokyo University of Agriculture

and Technology, Fuchu, Tokyo 183-8509, Japan

e-mail: ymatsu@cc.tuat.ac.jp plants. The results suggested that many ERF genes encoding EAR motif-containing proteins might have the ability to induce cell death when overexpressed.

Keywords TMV $\cdot N$ gene $\cdot$ Hypersensitive response . HR cell death $\cdot \mathrm{NtERF} 3 \cdot$ EAR motif

\section{Introduction}

Because plants are continuously exposed to environmental changes and stresses and unable to escape the stress, it is important for them to respond to external stimuli in a timely fashion. Plants have evolved many plant-specific transcription factors to precisely regulate the expression of their genes. Among them, the AP2/ERF superfamily is one of the largest families of transcription factors in plants (Nakano et al. 2006; Zhang et al. 2008; Zhuang et al. 2011). Ethylene response factors (ERFs) bind with high affinity to the conserved promoter sequence, called the GCC-box through the highly conserved AP2/ERF domain (Hao et al. 1998; Ohme-Takagi and Shinshi 1995). The GCC-box is a short conserved cis-element found in the promoter regions of several ethylene-responsive genes, such as the gene for basic PR protein (Ohme-Takagi et al. 2000; Shinshi et al. 1995). There have been many reports of the involvement of ERFs in responses to biotic and abiotic stresses as well as in plant hormone signaling. ERFs are also reported to be involved in resistance against pathogens. Examples include an increase or a decrease in disease resistance in $E R F$-expressing transgenic plants (Berrocal-Lobo et al. 2002; Bethke et al. 2009; Century et al. 2008; Fischer and Dröge-Laser 2004; Gu et al. 2002; McGrath et al. 2005; Park et al. 2001; Zhang et al. 2009). ERFs seem to have important functions in transcriptional 
regulation of various responses to biotic or abiotic stresses (Century et al. 2008; Nakano et al. 2006).

Plants have evolved resistance $(R)$ genes in response to biotic stresses such as infection by pathogens. $R$ gene products recognize elicitors derived from pathogens and induce host resistance processes such as hypersensitive response (HR) (Jones and Dangl 2006; Soosaar et al. 2005). The $N$ gene of Nicotiana tabacum (tobacco) is one of the TIR-NBS-LRR class of $R$ genes (Whitham et al. 1994). When tobacco harboring the $N$ gene ( $N N$ tobacco) is infected with Tobacco mosaic virus (TMV), the helicase domain (p50) of TMV replicase is recognized as an elicitor capable of inducing HR cell death, and under permissive temperatures, the viruses are confined to restricted necrotic areas. Some genes involved in the signal transduction pathway of HR cell death have the ability to induce HR-like cell death when ectopically overexpressed (Menke et al. 2005; Yang et al. 2001; Zhang and Liu 2001). Among ERF family genes, NbCD1 gene from Nicotiana benthamiana was reported to induce HR-like cell death when overexpressed in $N$. benthamiana and Arabidopsis thaliana (Nasir et al. 2005). NbCD1 is a transcriptional repressor that has a short, conserved ERFassociated amphiphilic repression (EAR) motif at its C-terminal region and is in the same class as tobacco NtERF3 and Arabidopsis AtERF3 and AtERF4 (Nasir et al. 2005). NtERF3 is one of four tobacco genes initially identified as ERFs (Ohme-Takagi and Shinshi 1995) and encodes an EAR motif-containing repressor protein (Ohta et al. 2000, 2001). The expression of NtERF3 is upregulated rapidly and transiently by ethylene or salicylic acid treatment or by wounding at the transcriptional level (Nishiuchi et al. 2002, 2004; Suzuki et al. 1998). Because it is important to suppress the expression of genes when their product is not required, transcriptional repression of expression of other genes by repressor proteins such as NtERF3 might play a key role in modulating plant stress responses (Kazan 2006). Although there have been some reports on the involvement of NtERF3 in stress responses, whether NtERF3 is involved in the signal transduction pathway to HR cell death is unknown. Though NbCD1 is in the same class as NtERF3, regions outside the conserved domains and motifs are quite different from each other, and the amino acid identity between entire proteins is not very high (45\%) (Nasir et al. 2005). This suggests that NtERF3 is not an orthologue of $N b C D 1$. Here, we report on the analysis of NtERF3 function in HR-mediated cell death. We first characterized the expression of $N t E R F 3$ during HR induction by TMV infection or ectopic expression of TMV $p 50$ in $N N$ tobacco. Then, we examined whether overexpression of NtERF3 or its homologues from tobacco, $N$. benthamiana and rice would induce HR-like cell death in tobacco plants.

\section{Materials and methods}

Plant growth conditions and TMV treatment

Tobacco plants (N. tabacum cv. Samsun NN and nn) and $N$. benthamiana plants were grown on Rockfiber blocks (Nittobo, Tokyo, Japan) at $25^{\circ} \mathrm{C}$ with a $16 \mathrm{~h} \mathrm{light} / 8 \mathrm{~h}$ dark photoperiod for 2 weeks. Seedlings were then transferred to pots filled with vermiculite and grown in the same conditions with $0.1 \%(\mathrm{v} / \mathrm{v})$ Hyponex fertilizer solution (Hyponex Japan, Osaka, Japan) added once a week. Plants at 8- to 10-weeks-old after sowing were used for all experiments. Oryza sativa cv. Nipponbare plants were grown as described previously (Hirabayashi et al. 2004).

For the induction of HR from the $N$ gene, $25 \mu$ l of $100 \mathrm{ng} / \mu \mathrm{l}$ virion of the TMV-OM strain was inoculated onto leaves of $N N$ tobacco mechanically with carborundum. The inoculated plants were incubated at $30^{\circ} \mathrm{C}$ for $40 \mathrm{~h}$ then all plants were shifted to $20^{\circ} \mathrm{C}$ to synchronously induce HR. Nonresistant $n n$ tobacco plants were used as non-HR controls. As a control, the same volume of $10 \mathrm{mM}$ sodium phosphate buffer ( $\mathrm{pH}$ 7.0) was used as a mock inoculation solution.

\section{Cloning of NtERF3b, NbERF3, NtERF6 $a$ and NtERF6b}

$N t E R F 3 b, N t E R F 6 a$ and NtERF6b were cloned from $N$. tabacum cv. Samsun NN while NbERF3 was cloned from $N$. benthamiana. Details of the cloning process are described in supplementary materials. Nucleotide sequences of $N t E R F 3 b, N b E R F 3, N t E R F 6 a$ and NtERF $6 b$ were submitted to DDBJ under the accession numbers AB573716, AB573717, AB573718 and AB573719, respectively.

\section{Construction of plasmids}

The details of construction of plasmids used in this study are described in the supplementary materials.

RNA isolation and reverse transcription-polymerase chain reaction (RT-PCR) analysis

Total RNA was isolated using the RNAiso reagent (Takara BIO, Shiga, Japan) according to the manufacturer's instructions. cDNA was synthesized from total RNA with Oligo dT Primer and Random 6-mers using PrimeScript RT Reagent Kit (Takara BIO). Semi-quantitative expression analysis was performed by RT-PCR with GoTaq Green Master Mix (Promega, Madison, WI, USA). The cDNA for Actin was amplified as an internal control. Specific oligonucleotides for each gene were as follows: Actin (5'-GG GTTTGCTGGAGATGATGCT- ${ }^{\prime}$ and $5^{\prime}$-GCCTTTGCAA TCCACATCTGTTG- $\left.3^{\prime}\right), H s r 203 J\left(5^{\prime}\right.$-CACCCCTATCGG 
AGCAAATCGGAG-3' and 5'-GATGAACTCTGCAACG GCTTC- $\left.3^{\prime}\right)$, Hin- 1 (5'-CACCGACCGATGCTACGTTG ACTC- $3^{\prime}$ and $5^{\prime}$-CTACCAATCAAGATGGCATCTG- $3^{\prime}$ ), acidic $P R-1$ (5'-TAGTCATGGGATTTGTTCTC-3' and $5^{\prime}$-TCAGATCATACATCAAGCTG- $3^{\prime}$ ), and basic $P R-1$ (5'-GGGATACTCCACAACATTAG- $3^{\prime}$ and 5'-CACA TACATATACACACCTC- $3^{\prime}$ ). Real-time quantitative PCR analysis was performed with the Smart Cycler II system (Takara BIO) using SYBR Premix Ex Taq (Takara BIO). The cDNA for Actin was amplified as an internal control to normalize the expression of each gene. The following oligonucleotides were used to detect the expression of each gene: Actin (5'-GGGTTTGCTGGAGATGATGCT- $3^{\prime}$ and $5^{\prime}$-GCTTCGTCACCAACATATGCAT-3'), NtERF3 (5'-A GGAATTGATCTTGATCTTAAC- $3^{\prime}$ and $5^{\prime}$-ACAAAAT TCAACCATTAGTCTC- $\left.3^{\prime}\right), N t W R K Y 1$ (5'-GATGTTACA GAGCTCTGGAAATTC- $3^{\prime}$ and $5^{\prime}$-TCGGCTTGATAT TATTCATGGGC- $\left.3^{\prime}\right)$, and $P R-1 b\left(5^{\prime}\right.$-CAATAGGTTAGC GGCCTTTG- $3^{\prime}$ and $5^{\prime}$-TTGCTTCTCATCAACCCACA-3 ${ }^{\prime}$ ).

\section{Agrobacterium infiltration}

Agrobacterium tumefaciens strain GV3101 (pMP90) was transformed with expression plasmids and tobacco leaves were infiltrated with bacteria essentially as described previously (Sasaki et al. 2009). The final concentrations of the bacteria were adjusted to an $\mathrm{OD}_{600}$ of 0.4 with infiltration buffer containing $10 \mathrm{mM} \mathrm{MES,} \mathrm{pH} \mathrm{5.7,} 10 \mathrm{mM} \mathrm{MgCl}_{2}$ and $150 \mu \mathrm{M}$ acetosyringone. The plants were kept at $20^{\circ} \mathrm{C}$ after Agrobacterium infiltration.

Determination of ion leakage

Leaf materials were excised from the infiltrated plants and soaked in $\mathrm{H}_{2} \mathrm{O}$ at a ratio of $1 \mathrm{ml} \mathrm{H}_{2} \mathrm{O}$ per $1 \mathrm{~cm}^{2}$ of leaf materials. After incubation at $20^{\circ} \mathrm{C}$ for the indicated duration, conductivity of the water was measured using a conductivity meter (model B-173, Horiba, Kyoto, Japan) and was expressed as $\mu \mathrm{S} \mathrm{cm} \mathrm{cm}^{-1} \mathrm{~cm}^{-2}$ of leaf materials.

Measurement of reactive oxygen species (ROS) generation

ROS were detected using 8-amino-5-chloro-7-phenylpyrido $[3,4-d]$ pyridazine-1,4- $(2 H, 3 H)$ dione sodium salt (L-012) (Wako Chemical, Kanagawa, Japan). Tobacco leaves were infiltrated with L-012 solution (0.5 mM L-012, $10 \mathrm{mM}$ MOPS-KOH, $\mathrm{pH}$ 7.4) using a needleless syringe to fill the Agrobacterium-infiltrated areas. After $5 \mathrm{~min}$, chemiluminescence of the L-012-infiltrated leaves was measured for $5 \mathrm{~min}$ using LAS-3000 system (FujiFilm, Tokyo, Japan). The intensity of the chemiluminescence was converted into numerical values using MultiGauge ver.
2.2 analysis software (FujiFilm). The relative intensity per unit area of the Agrobacterium-infiltrated region was calculated after the subtraction of the background fluorescence value of the non-Agrobacterium-infiltrated regions from the treated leaves.

Recombinant virus inoculation

Template plasmids pTogJ-NtERF3b $\Delta$ EAR-DHA, pTogJNtERF3b-DHA and the control plasmid pTocJ-GFP (Hori and Watanabe 2003) were digested with $M l u \mathrm{I}$ and purified with UltraClean15 DNA Purification Kit (MO BIO Laboratories, Carlsbad, CA, USA). One microgram of the purified DNAs was subjected to in vitro transcription in a volume of $25 \mu \mathrm{l}$ with T7 RNA polymerase as described (Hori and Watanabe 2003). Leaves of $n n$ tobacco and $N$. benthamiana were mechanically inoculated with $8 \mu \mathrm{l}$ of the transcripts using carborundum.

Imaging by fluorescence microscopy

GFP fluorescence in Agrobacterium-infiltrated leaf tissues was observed with a BX50 fluorescence microscope equipped with a UMWIBA/GFP filter unit (OLYMPUS, Tokyo, Japan). For staining the nucleus, leaves that were sampled at $32 \mathrm{~h}$ after Agrobacterium infiltration were infiltrated with $10 \mu \mathrm{g} / \mathrm{ml}$ of $4^{\prime}, 6$-diamidino-2-phenylindole (DAPI). After incubation for $30 \mathrm{~min}$ to $1 \mathrm{~h}$, the DAPIstained leaves were observed with the fluorescence microscope and filter unit U-MWU (OLYMPUS). Images were taken with a DP71 digital camera (with DP Controller ver. 3.1.1 and DP Manager ver. 3.1.1, OLYMPUS).

\section{Results}

Expression of $N t E R F 3$ is upregulated during HR cell death induction by $N$ gene

Nicotiana tabacum is thought to be an amphiploid derived from Nicotiana sylvestris and Nicotiana tomentosiformis (Murad et al. 2002). Therefore, it was thought that the tobacco genome contained two types of NtERF3 genes, one from each ancestor. To characterize the expression of NtERF3 during HR induction, we first isolated each type of cDNA for NtERF3 from $N$. tabacum by utilizing DNA sequence information for NtERF3 (DDBJ accession D38124) from $N$. tabacum and NsERF3 (DDBJ accession AB016265) from $N$. sylvestris. One type of cDNA was $100 \%$ identical to NtERF3 (DDBJ accession D38124) at the nucleotide level, while the other type was almost identical to NsERF3 with a single-base difference in the protein-coding region. To distinguish the two types of 
$N t E R F 3$, a novel $N$. sylvestris-type of NtERF3 was designated as NtERF3b (deposited in DDBJ as accession AB573716), and the other type of NtERF3 (DDBJ accession D38124) called NtERF3a in this work. The deduced amino acid sequences had a single-amino acid difference between NtERF3b and NsERF3, and 16 amino acids differing (94\% identity) between NtERF3a and NtERF3b, including the insertion of two amino acids in NtERF3b. On the basis of the nucleotide sequence data, a set of oligonucleotides was prepared to amplify both NtERF3a and NtERF3b.

For HR induction, we used a combination of TMV and its specific resistance gene $N$ as a model system. The HR controlled by the $N$ gene is temperature-dependent and does not occur under nonpermissive temperatures. This feature is often exploited to uniformly and synchronously induce HR in several samples (Takabatake et al. 2007). Using a synchronous HR-induction system based on a temperature shift from $30^{\circ} \mathrm{C}$, a nonpermissive temperature for the $N$ gene, to $20^{\circ} \mathrm{C}$, a permissive temperature, we investigated the expression level of NtERF3 during $\mathrm{HR}$ induction. In the $N$ gene-containing $N N$ tobacco infected with TMV, cell death lesions became visible from 5 to $7.5 \mathrm{~h}$ after the temperature shift (data not shown), and ion leakage, an indicator of cell death, also increased from 5 to $7.5 \mathrm{~h}$ after the temperature shift (Fig. 1a). In contrast, no cell death was observed, and ion leakage did not significantly change in either TMVinfected $n n$ tobacco or mock-inoculated $N N$ tobacco (Fig. 1a). Gene expression of NtERF3 was upregulated significantly from 2.5 to $5 \mathrm{~h}$ after the temperature shift, and this increased expression state lasted during the cell death progression only in TMV-infected $N N$ tobacco (Fig. 1b).

The expression levels of $N t W R K Y 1$ and $P R-1 b$ genes were also examined because it was known that both genes were upregulated during HR and that the promoter region of NsERF3 contains a cis-element, the W-box, to which NtWRKY1 binds in vitro (Nishiuchi et al. 2004). Both genes were upregulated during the progression of cell death only in the TMV-infected $N N$ tobacco, as in the case of NtERF3 (Fig. 1c, d). Upregulation of $N t W R K Y 1$ was comparable to that of NtERF3, while $P R$ $1 b$ was upregulated later than NtERF3 (Fig. 1b-d). Agrobacterium-mediated ectopic expression of the TMV $p 50$, an elicitor to the $N$ gene, is also known to induce HR cell death in $N N$ tobacco (Erickson et al. 1999). We used this Agrobacterium-infiltration method for the induction of HR. In this system, cell death lesions became visible from 36 to $48 \mathrm{~h}$ after the infiltration of $p 50$ (data not shown). The mRNA level of NtERF3 in the p50-expressing $N N$ tobacco was higher at $32 \mathrm{~h}$ after
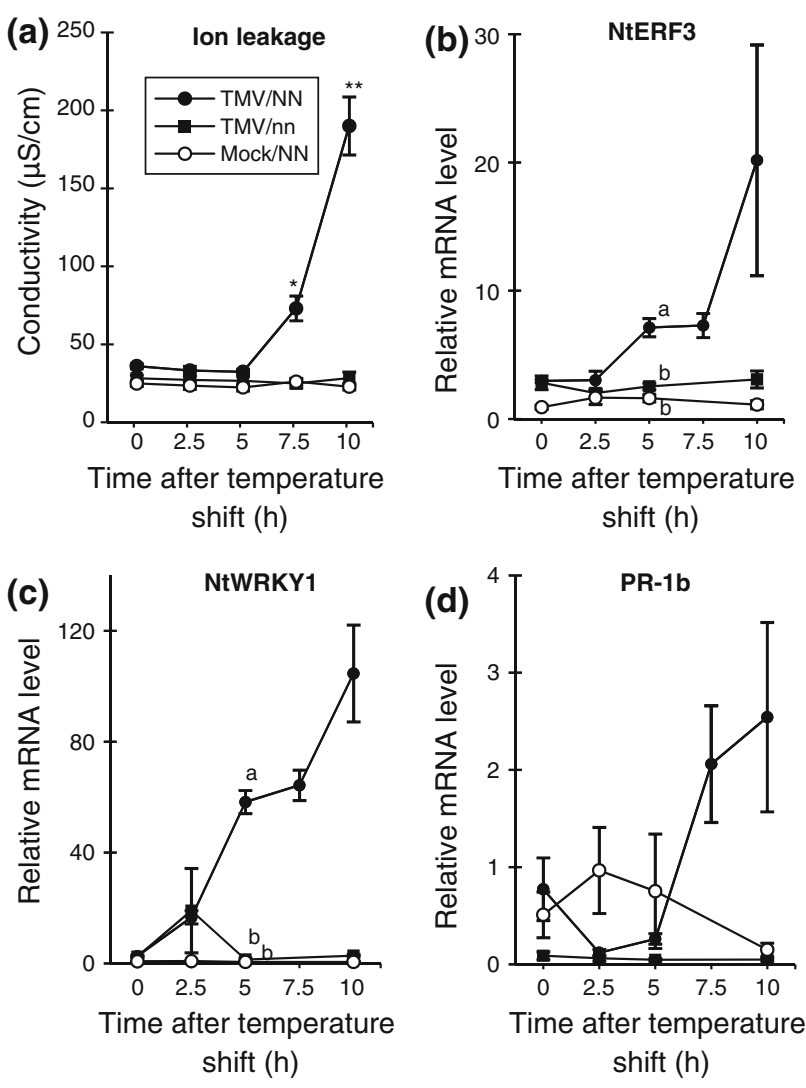

Fig. 1 Upregulation of NtERF3 during hypersensitive response induction by the $N$ gene. a Ion leakage from tobacco leaves inoculated with Tobacco mosaic virus (TMV) or buffer (TMV/NN, $\mathrm{TMV} / \mathrm{nn}$ and Mock/NN) after the temperature shift from 30 to $20^{\circ} \mathrm{C}$. Leaf materials were excised at the indicated time points after the temperature shift, soaked in water for $30 \mathrm{~min}$, and conductivity of the water was measured. Values are presented as mean $\pm \operatorname{SE}(n=6)$, and significant differences between data at each time point with respect to $0 \mathrm{~h}$ were assessed with Dunnett's test; ${ }^{*} P<0.05$, $* * P<0.01$. Black and white circles in a-d indicate TMV/NN and Mock/NN, and black squares indicate TMV/nn, respectively. Realtime PCR analysis of NtERF3 (b), NtWRKY1 (c) and PR-1b (d) expression at the indicated time points after the temperature shift. Values are mean $\pm \mathrm{SE}(n=6)$ relative to mRNA levels in untreated $N N$ tobacco (untreated $N N$ tobacco; set arbitrarily at 1). Relative mRNA levels were normalized to the expression of Actin gene measured in the same samples. Different letters $(a, b)$ at $5 \mathrm{~h}$ indicate significant differences among the three plants as determined by Tukey's test $(P<0.01)$

infiltration than that in the control $s G F P$-expressing or mock-infiltrated $N N$ tobacco, in which HR had not been induced (Fig. S1a). NtERF3 was known to be one of the wound-induced genes whose expression was induced rapidly and suppressed to the normal level soon afterward (Nishiuchi et al. 2002, 2004; Suzuki et al. 1998). The expression levels of NtERF3 in the wound-treated $N N$ tobacco increased about 30 -fold by $30 \mathrm{~min}$ following treatment relative to the untreated control tobacco (Fig. S1b). 
Overexpression of NtERF3 induces HR-like cell death in tobacco

$N t E R F 3 a$ and NtERF3b were transiently overexpressed in tobacco treated by the Agrobacterium-infiltration method. Overexpression of NtERF3a and NtERF3b induced cell death, just as did the TMV elicitor (p50) in $N N$ tobacco (Fig. 2a, NtERF3a, NtERF3b and p50). Addition of an HAtag at the C-terminus did not affect cell death induction (NtERF3a::HA and NtERF3b::HA). In contrast, deletion of the EAR motif from NtERF3b (NtERF3b- $\triangle E A R:$ HA) resulted in no induction of cell death, just as with inoculation with sGFP, empty vector (Empty vector) or mock infiltration (Mock). Similar results were obtained in $n n$ tobacco, in which, due to the absence of $N$ gene, p50 did not induce cell death (Fig. S2a). Overexpression of $N t E R F 3 a$ and NtERF3b in N. benthamiana also induced cell death, although the manifestation of cell death was not as clear as that in tobacco (data not shown). HA-tagged proteins (NtERF3a::HA, NtERF3b::HA and NtERF3b- $\triangle$ EAR::HA) became detectable at $24 \mathrm{~h}$ following infiltration into $N N$ tobacco, and their level continued to increase (Fig. S2b), while cell death lesions became visible from 36 to $48 \mathrm{~h}$ after the infiltration of NtERF3a::HA- or NtERF3b::HA-expressing Agrobacterium (data not shown). It is known that HR cell death is associated with the production of reactive oxygen species (ROS) and an increase in ion leakage (Jones and Dangl 2006; Soosaar et al. 2005; Takabatake et al. 2007). Cell death induced by overexpression of NtERF3s was associated with an increase of ion leakage (Fig. 2b) and ROS production (Fig. 2c), as in the case of HR cell death induced by the TMV $p 50$ in $N N$ tobacco. In addition, expression of marker genes for the HR was upregulated in the Agrobacteriuminfiltrated regions of $N t E R F 3 \mathrm{a}$ - and $p 50$-treated leaves, but not in those of $s G F P$-treated leaves (Fig. 2d).

To further examine the ability of NtERF3 to induce cell death, we used a virus vector, pTogJ (Hori and Watanabe 2003), as another system for ectopic gene expression. The recombinant virus RNA encoding NtERF3b (Fig. 3a, b) (a)

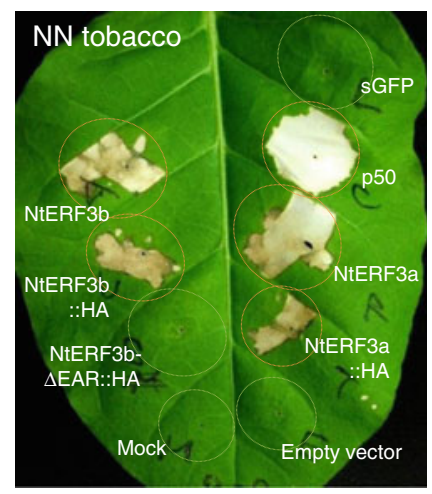

(c)
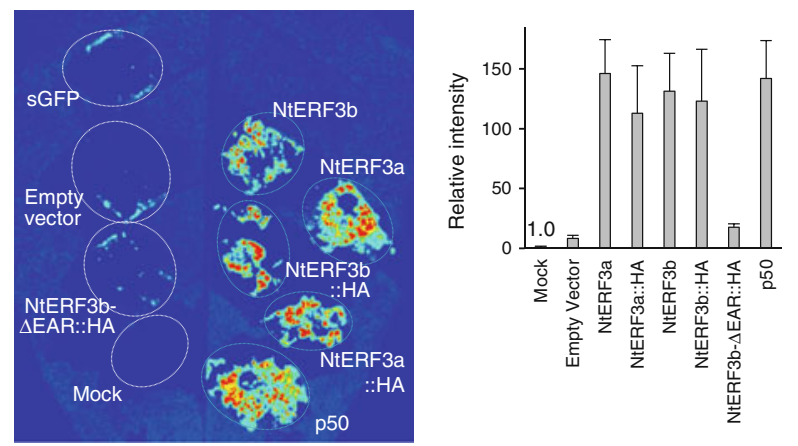

(d)

(b)


Fig. 2 Hypersensitive response (HR)-like cell death induction by Agrobacterium-mediated transient overexpression of NtERF3 in tobacco. a NtERF3a, NtERF $3 b$ and their HA-tag-fused derivatives were transiently expressed in $N N$ tobacco by Agrobacterium infiltration. Empty vector, $s G F P$ and the infiltration buffer (Mock) were used as negative controls. The helicase domain of Tobacco mosaic virus (TMV) replicase ( $p 50)$, the elicitor to the $N$ gene, was used as a positive control for induction of HR cell death. The photograph was taken at 7 days after infiltration. Circles generally indicate each infiltrated area. b Ion leakage from tobacco leaves at $48 \mathrm{~h}$ after Agrobacterium infiltration. Leaf materials were excised and soaked in water for $4 \mathrm{~h}$, and conductivity of the water was measured. Values are mean $\pm \operatorname{SE}(n=5)$. c Production of reactive oxygen species (ROS) in $N N$ tobacco leaves at $32 \mathrm{~h}$ after Agrobacterium infiltration. The image of chemiluminescence due to ROS production is shown on the left panel. Circles generally indicate each infiltrated area. Relative intensities of chemiluminescence at each infiltrated area are shown on the right graph. Values are mean $\pm \mathrm{SE}(n=5)$ relative to the value of mock-infiltrated region (Mock; set arbitrary at 1). d RT-PCR analysis of the expression of Hin-1, Hsr203J and basic PR-1 in NN tobacco at $42 \mathrm{~h}$ after Agrobacterium infiltration. Expression of Actin was investigated as a control 
induced cell death lesions in the leaves of $n n$ tobacco and $N$. benthamiana, while those encoding NtERF3b without the EAR motif (Fig. 3d, e) and GFP (Fig. 3g, h) did not induce cell death. In $n n$ tobacco and $N$. benthamiana inoculated with the NtERF3b-encoding recombinant virus RNA, cell death lesions became visible at 3-4 days after inoculation, while in those inoculated with GFP-encoding recombinant virus RNA, GFP fluorescence was confirmed at 3-4 days after inoculation (data not shown). Because pTogJ virus vector encodes TMV replicase, the recombinant viruses are able to induce $N$ gene-mediated HR cell death in $N N$ tobacco. This was utilized to confirm the infection of each recombinant virus in $n n$ tobacco. Crude extracts prepared from the leaves of $n n$ tobacco inoculated with each recombinant virus RNA (Fig. 3a, d, g) were secondarily inoculated to $N N$ tobacco. Such inoculation resulted in the appearance of cell death lesions (Fig. 3c, f, i). Expression of the marker genes for HR was upregulated in the leaves inoculated with the NtERF3b-encoding recombinant virus RNA, but was not in those inoculated with the GFP-encoding recombinant virus RNA (Fig. 3j).

EAR motif of $N t E R F 3$ is essential for cell death induction but not for NtERF3 subcellular site

The EAR motif has been shown to function as a repression motif in some ERF proteins and $\mathrm{C} 2 \mathrm{H} 2$ zinc-finger proteins (Ohta et al. 2001). In Figs. 2 and 3, the deletion of the EAR motif from NtERF3b was shown to affect the induction of cell death. Although it was previously reported that NtERF3 localized to nuclei in tobacco BY-2 cell (Ohta et al. 2000), the influence of its EAR-motif on subcellular localization has not been comprehended. To examine whether deletion of the EAR motif would affect the subcellular localization of the NtERF3 protein, expression plasmids for the sGFP-fused proteins NtERF3b::sGFP and NtERF3b- $\triangle$ EAR::sGFP were constructed and transiently expressed in $N N$ tobacco by the Agrobacterium-infiltration method. At $32 \mathrm{~h}$ after Agrobacterium infiltration, GFP fluorescence was observed exclusively in the nuclei of the NtERF3b::sGFP- and NtERF3b$\triangle$ EAR::sGFP-producing cells, but not in those of the in sGFP-producing cells (Fig. 4a). In addition, attachment of the EAR motif to the C-terminus of sGFP had no effect on the subcellular localization of the protein (Fig. 4b). Cell death lesions became visible in the Agrobacterium-infiltrated area of NtERF3b::sGFP leaves, but not in those of NtERF3b$\triangle$ EAR::sGFP nor sGFP leaves at $36 \mathrm{~h}$ after the infiltration (data not shown), and the cell death lesions induced by NtERF3b::sGFP became more distinct (Fig. 4c). Reattachment of the EAR motif to the C-terminus of the EAR motifdeleted mutant restored the ability of the protein to induce cell death (Fig. 4d, NtERF3b- $\triangle$ EAR::EAR::HA). When the EAR motif was fused to the wild type NtERF3b, the cell
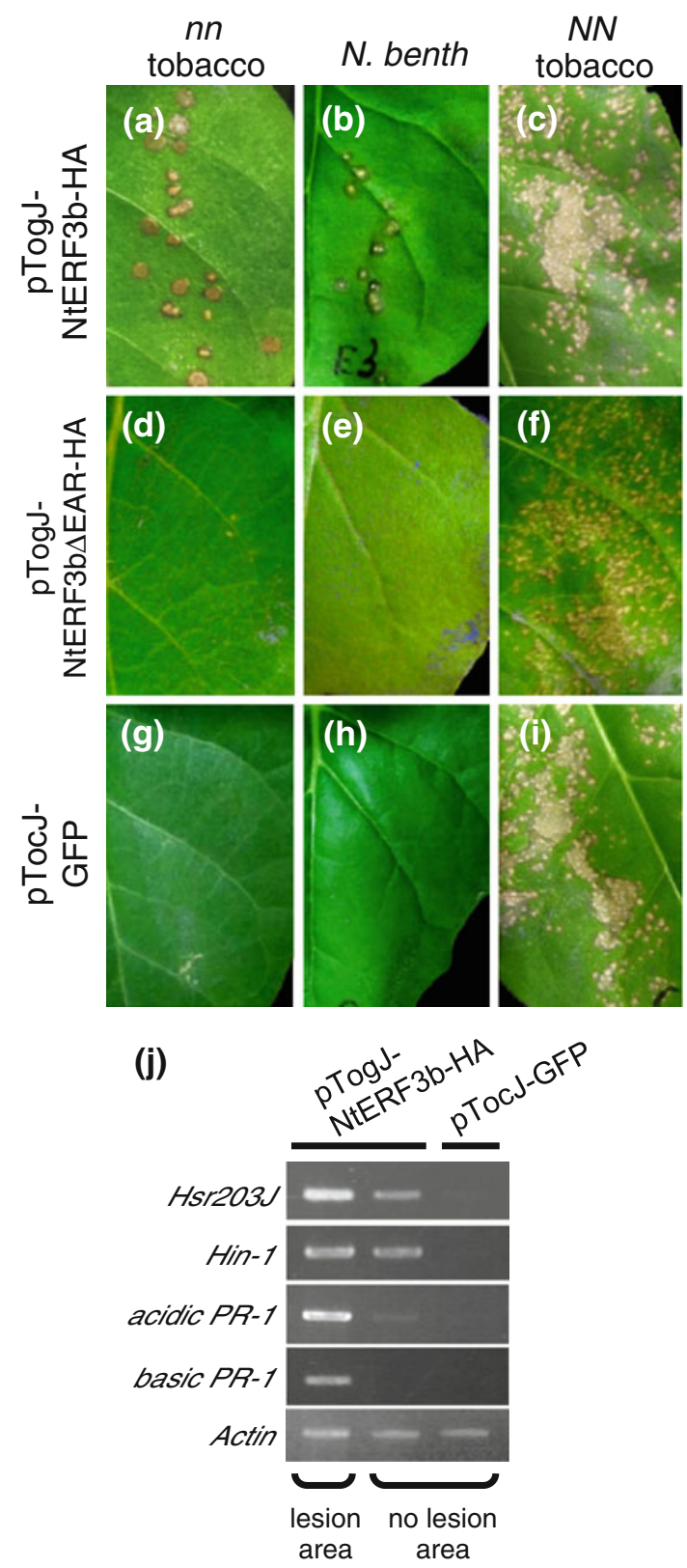

Fig. 3 Hypersensitive response-like cell death induction by virus vector-mediated overexpression of NtERF3. Leaves of $n n$ tobacco $(\mathbf{a}, \mathbf{d}, \mathbf{g})$ and Nicotiana benthamiana $(N$. benth) $(\mathbf{b}, \mathbf{e}, \mathbf{h})$ were inoculated with in vitro transcripts derived from pTogJ-NtERF3bDHA (a, b), pTogJ-NtERF3b $\Delta E A R-H A(\mathbf{d}, \mathbf{e})$ and pTocJ-GFP $(\mathbf{g}, \mathbf{h})$. Crude extracts prepared from inoculated leaves of $n n$ tobacco were secondarily inoculated to $N N$ tobacco $(\mathbf{c}, \mathbf{f}, \mathbf{i})$. Photographs were taken at 7 or 6 days after the inoculation of tobacco or $N$. benthamiana, respectively. j Expression analysis of Hsr203J, Hin-1, acidic PR-1 and basic $P R-1$ by RT-PCR in $n n$ tobacco at 7 days after the inoculation of in vitro transcripts from TogJ plasmids. For pTogJNtERF3b-HA, leaf materials were prepared from areas with (lesion area) or without (no lesion area) cell death lesions. Expression of Actin was examined as a control

death-inducing ability of the resultant protein NtERF3b::EAR::HA was comparable to that of NtERF3b::HA (Fig. 4d). In contrast, the ectopic expression of the EAR 


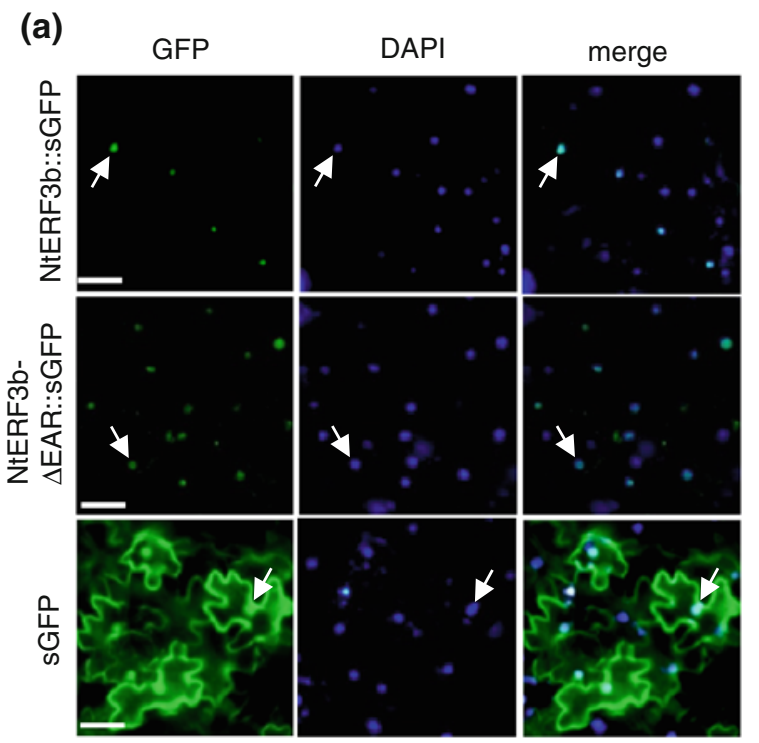

size bar $=100 \mu \mathrm{m}$ (b)

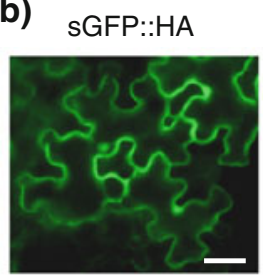

SGFP::EAR::HA

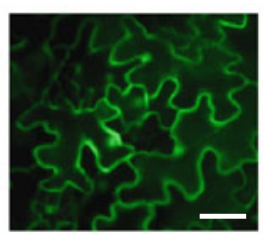

size bar $=50 \mu \mathrm{m}$ (c)

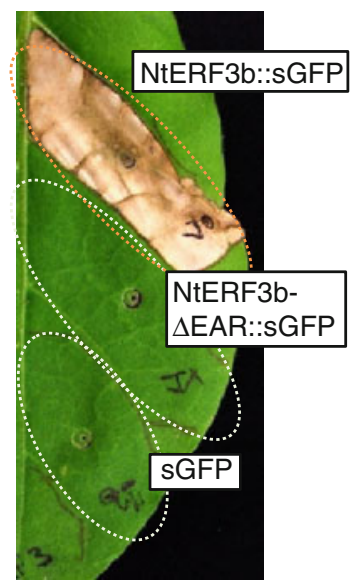

(d)

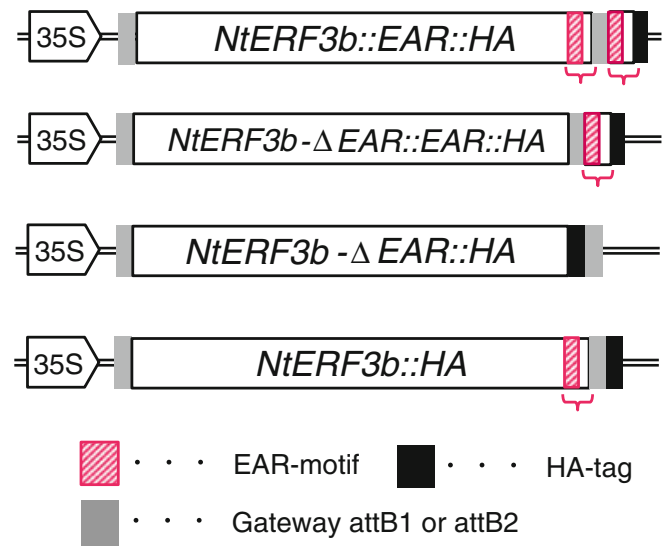

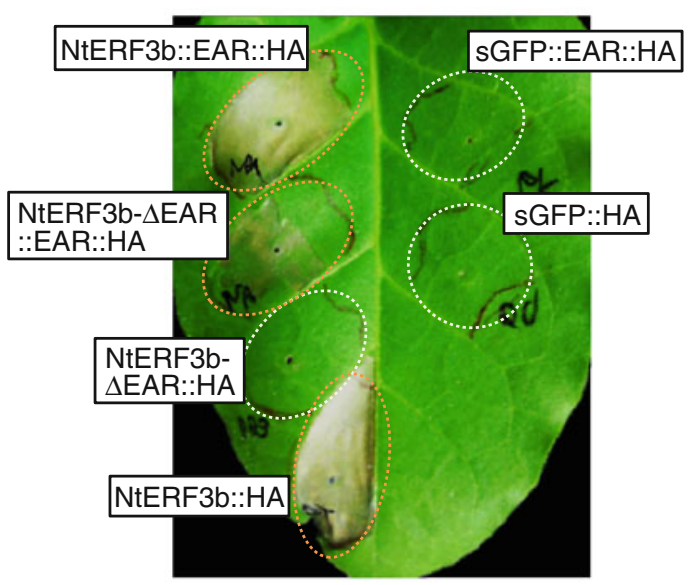

Fig. 4 Nuclear localization and cell death-inducing ability of the EAR motif-deleted NtERF3. a NtERF3b::sGFP, NtERF3b$\triangle E A R:: s G F P$ or $s G F P$ were transiently overexpressed in tobacco leaves by Agrobacterium infiltration. GFP and DAPI fluorescence were observed by microscopy at $32 \mathrm{~h}$ after the infiltration. Both fluorescent images are merged in the third row (merge). White arrows indicate one of the images for nuclei showing the fluorescence of GFP or DAPI. b $s G F P: \because H A$ and $s G F P: \because E A R: \because H A$ were expressed in tobacco leaves, and GFP fluorescence was observed at 3 days after

motif-fused (sGFP::EAR::HA) or non-fused sGFP (sGFP::HA), as well as the EAR motif-deleted NtERF3b (NtERF3b- $\triangle \mathrm{EAR}:: \mathrm{HA}$ ) induced no visible change at the Agrobacterium-infiltrated area at 3 days after the infiltration (Fig. 4d).

NtERF3 homologues from tobacco, $N$. benthamiana and rice induce cell death in tobacco

The deduced amino acid sequences of NtERF3a and $\mathrm{NbCD} 1$ were highly conserved in the AP2/ERF domain

Agrobacterium infiltration. c Photograph of leaf in a was taken at 7 days after the infiltration. d NtERF3b::EAR::HA, NtERF3b$\triangle E A R:: E A R:: H A, \quad N t E R F 3 b-\triangle E A R:: H A, \quad$ NtERF3b::HA, sGFP:: EAR::HA or $s G F P:: H A$, were overexpressed by Agrobacterium infiltration. Photograph was taken at 3 days after the infiltration. Schematic drawing of plasmid constructs is on the left. Boxes with slanted lines depict the EAR motifs, while black and gray boxes depict HA-tags and the additional amino acids derived from the attB Gateway recombination sequences, respectively

and EAR motif, but not in other regions (Fig. S3). Therefore, genes with higher identity to $N b C D 1$ than $N t E R F 3 a$ and $N t E R F 3 b$ were expected in the genome of $N$. tabacum, and vice versa for $N$. benthamiana. Such genes were isolated by RT-PCR using the oligonucleotides designed based on the nucleotide sequence information of $N b C D 1$, $N t E R F 3 a$ and NtERF3b. Two kinds of NbCDI homologues were isolated from $N$. tabacum and named NtERF6a and $N t E R F 6 b$, respectively, while one type of NtERF3 homologue was isolated from $N$. benthamiana and named NbERF3. In contrast to the amino acid identity of NtERF3a 
and NtERF3b to NbCD1 (45 and 40\%, respectively), NtERF6a and NtERF6b were 89 and $89 \%$ identical to NbCD1, respectively, while NbERF3 was 93 and $92 \%$ identical to NtERF3a and NtERF3b, respectively (Fig. S3). When these newly isolated genes were transiently overexpressed in tobacco after Agrobacterium infiltration, HRlike cell death was induced, as in the case of NtERF $3 a$ and NbCDl (Fig. 5a, b). The results raised the question whether other $E R F$ genes encoding EAR motif-containing proteins would have the ability to induce cell death when overexpressed, because many ERF genes encoding the EAR motif-containing proteins have been reported for various plant species (Nakano et al. 2006; Ohta et al. 2001; Zhang et al. 2008). Two genes, OsERF\#074 (DDBJ accession AK111755) and OsERF\#075 (DDBJ accession AK069262, reported as OsERF3) were isolated from a monocotyledonous plant, $O$. sativa, based on nucleotide sequence information (Nakano et al. 2006). Transient overexpression of OsERF\#074 and OsERF\#075 by the Agrobacterium-infiltration method also induced HR-like cell death in tobacco (Fig. 5c).

\section{Discussion}

In this study, a tobacco transcriptional repressor NtERF3 was analyzed for its putative function in HR cell death. $N t E R F 3$ was cloned from two types of tobacco NtERF3 (NtERF3a and NtERF3b), one from each ancestor of amphiploid tobacco, N. sylvestris or $N$. tomentosiformis. (a)

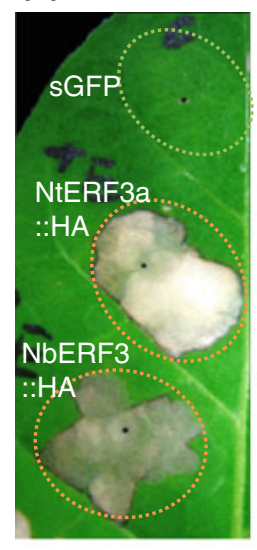

(b)

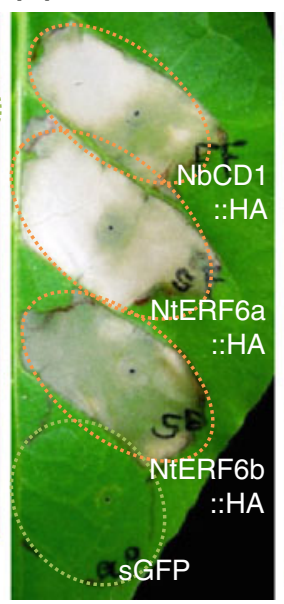

(c)

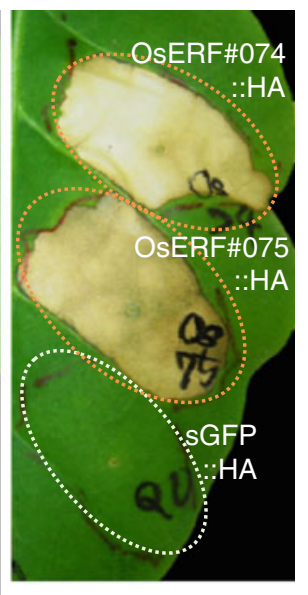

Fig. 5 Cell death induction by NtERF3 homologues. HA-tag-fused derivatives of Nicotiana benthamiana NbERF3 (a), tobacco NtERF6a and NtERF6b (b) and Oryza sativa OsERF\#074 and OsERF\#075 (c) were transiently expressed in $N N$ tobacco leaves by Agrobacterium infiltration. $s G F P$ and $s G F P:: H A$ were used as negative controls. Photographs were taken at 5 (b) or 7 days $(\mathbf{a}, \mathbf{c})$ after the infiltration, respectively
Transient overexpression of NtERF3a and NtERF3b induced cell death in tobacco following Agrobacterium infiltration (Figs. 2a, S2a), as well as by the direct inoculation of an NtERF3-containing recombinant virus RNA (Fig. 3a). The NtERF3-induced cell death could be described as HR-like cell death because it was associated with an increase in ion leakage (Fig. 2b), ROS production (Fig. 2c), and upregulation of marker genes for $\mathrm{HR}$ (Figs. 2d, 3j), as in the case of HR cell death induced by the TMV elicitor p50 in $N N$ tobacco. The EAR motif of the NtERF3 protein had some crucial role on its cell deathinducing ability though it had no effect on nuclear localization of NtERF3 (Figs. 2, 3, 4). In addition to demonstrating cell death-inducing ability of NtERF3, we showed that other EAR motif-containing ERFs from tobacco, $N$. benthamiana and rice also induced cell death when overproduced in tobacco plants (Fig. 5). These results suggest that many ERF genes encoding EAR motif-containing proteins have the ability to induce cell death when overexpressed and that the cell death-inducing processes downstream of NtERF3 are conserved among monocot and dicot plant species.

The alignment of amino acid sequences of the EAR motif-containing ERF proteins used in this work showed that the AP2/ERF domain in the middle region and the EAR motif (DLNxxP) in the C-terminal region were highly conserved (Fig. S3). The consensus sequence of the EAR motif was at first reported L/FDLNL/FxP in tobacco NtERF3, and in Arabidopsis AtERF3 and AtERF4 (Ohta et al. 2001). Recently, Kagale et al. (2010) reported two distinct conservation patterns of EAR motif (DLNxxP and LxLxL) and identified 219 EAR motif-containing proteins belonging to 21 different families in Arabidopsis. The LxLxL type of EAR motif was first reported in the SUPERMAN protein of Arabidopsis (Hiratsu et al. 2002). The ERF proteins shown in Fig. S4 also have the LxLxL type of EAR motif in their middle region, as well as in their C-terminal region with overlapping the DLNxxP type of EAR motif. Two serine-rich regions exist between the AP2/ERF domain and the acidic region (Fig. S3). Nasir et al. (2005) reported that the AP2/ERF domain, acidic region and EAR motif were essential for the cell deathinducing ability of $\mathrm{NbCD} 1$, but that the region between the AP2/ERF domain and the acidic region was not required. The LxLxL type of EAR motif and the serine-rich region in the middle area might have other functions than cell death induction.

There are many possible downstream target genes for NtERF3, which contain the GCC box in their promoter regions. Non-GCC box-containing promoters could also be the targets of NtERF3, as Chakravarthy et al. (2003) reported that tomato transcriptional activator Pti4, a homologue of tobacco NtERF2, regulated defense-related 
gene expression via non-GCC box cis element. Elucidation of the downstream target genes of NtERF3 required for the cell death induction will facilitate an understanding of the HR cell death-inducing pathway in tobacco.

It was reported that NtERF1 and NtERF2 were upregulated during the HR cell death induction from $N$ gene (Kim et al. 2003). In this study, NtERF3 was revealed to be upregulated during HR cell death induction by the $N$ gene (Figs. 1b, S1a). Before the cell death lesions became visible, NtERF3 had started to be upregulated in $N N$ tobacco at $0-5 \mathrm{~h}$ after the temperature shift to $20^{\circ} \mathrm{C}$, and the state of upregulation lasted during the cell death progression (Fig. 1b). However, the extent of the NtERF3 upregulation during HR cell death induction (Figs. 1b, S1a) was much lower than that induced by the wounding treatment (Fig. $\mathrm{S} 1 \mathrm{~b})$. It was reported that the mRNA level of NtERF3 by wounding became maximal about $1 \mathrm{~h}$ after the wounding treatment and decreased rapidly afterward (Nishiuchi et al. 2002, 2004; Suzuki et al. 1998). However, the upregulation of NtERF3 by wounding did not induce cell death. The long-lasting upregulation of NtERF3 might be important for HR cell death induction. From this point of view, it would be interesting to note that a salicylic acid-induced protein kinase, SIPK and a wounding-induced protein kinase, WIPK, were regulated in different manners depending on the stress. For example, the SIPK protein kinase activity was transient in the wound response, but lasted long in HR cell death induction (Zhang and Klessig 1998; Zhang and Liu 2001).

In addition to the upregulation of NtERF3 during HR cell death induction by the $N$ gene, we tried to show the effect of loss of function of NtERF3 on TMV- $N$ genemediated HR cell death. In transgenic $N N$ tobacco plants expressing antisense or inverted-repeat transcripts of NtERF3, cell death by TMV infection still occurred although there was a tendency that the size of the cell death lesions was larger than that in wild-type plants (Fig. S4). Because the AP2/ERF superfamily is one of the largest families of transcription factors in plants (Nakano et al. 2006), there may be functional redundancy in the cell death induction between NtERF3 and its homologous genes. Silencing such genes altogether may be effective to clearly demonstrate the involvement of $N t E R F 3$ in TMV- $N$ genemediated HR.

McGrath et al. (2005) reported that a transcriptional repressor AtERF4, an Arabidopsis homologue of NtERF3, functioned as a negative regulator in the expression of a jasmonate (JA)-responsive gene PDF1.2, one of the defense-related genes containing GCC-box in its promoter region. In contrast, a truncated-type of AtERF4 without the EAR motif acted as a positive regulator of $P D F 1.2$ in a T-DNA insertion mutant of AtERF4 (erf4-1). AtERF4overexpressing transgenic plants had decreased resistance to a necrotrophic fungal pathogen Fusarium oxysporum, while erf4- 1 mutant plants had increased resistance in compared to the wild-type plants (McGrath et al. 2005). The involvement of AtERF4 in the basal disease resistance to pathogens may suggest that NtERF3 could be concerned with multiple processes such as the HR and basal disease resistance. Analysis of NtERF3 using pathogens other than TMV will provide new insight into the roles of NtERF3 in many biological processes, including the defense responses in tobacco. Further studies of NtERF3 and its homologues in other plant species, such as Arabidopsis and rice, will contribute to a better understanding of the defense responses in plants.

Acknowledgments We thank Drs. Nobumitsu Sasaki, Hiroshi Nyunoya and Ichiro Mitsuhara for valuable discussion and encouragement, Dr. Yasuo Niwa for plasmid p35 $\Omega$-sGFP(S65T), Dr. Toru Fujiwara for pTF336, Dr. Yuichiro Watanabe for pTocJ-GFP and pTogJ, Dr. Ko Shimamoto for pANDA35HK. We are grateful to Drs. Masahiro Kasahara, Nobumitsu Sasaki and Hiromi Kanegae for the construction of plasmids pART7-DHA, pART27-35Sa-GWB and pART27-35Sa-GWB-DHA, respectively. We also thank Chika Tachibana for the assistance with the early steps of NtERF3 cloning. This work was supported in part by a Grant-in-Aid for Scientific Research (C) (to Y.M., no. 19580045) from the Japan Society for the Promotion of Science (JSPS) and a Grant-in-Aid for JSPS Fellows (to T.O., no.23-8011), as well as research grants (to Y.M.) from the Collaborative Research Ring of Tokyo University of Agriculture and Technology (TUAT) and the TUAT Foundation.

Open Access This article is distributed under the terms of the Creative Commons Attribution Noncommercial License which permits any noncommercial use, distribution, and reproduction in any medium, provided the original author(s) and source are credited.

\section{References}

Berrocal-Lobo M, Molina A, Solano R (2002) Constitutive expression of ETHYLENE-RESPONSE-FACTOR1 in Arabidopsis confers resistance to several necrotrophic fungi. Plant J 29:23-32

Bethke G, Unthan T, Uhrig JF, Pöschl Y, Gust AA, Scheel D, Lee J (2009) Flg22 regulates the release of an ethylene response factor substrate from MAP kinase 6 in Arabidopsis thaliana via ethylene signaling. Proc Natl Acad Sci USA 106:8067-8072

Century K, Reuber TL, Ratcliffe OJ (2008) Regulating the regulators: the future prospects for transcription-factor-based agricultural biotechnology products. Plant Physiol 147:20-29

Chakravarthy S, Tuori RP, D'Ascenzo MD, Fobert PR, Després C, Martin GB (2003) The tomato transcription factor Pti4 regulates defense-related gene expression via GCC box and non-GCC box cis elements. Plant Cell 15:3033-3050

Erickson FL, Holzberg S, Calderon-Urrea A, Handley V, Axtell M, Corr C, Baker B (1999) The helicase domain of the TMV replicase proteins induces the $N$-mediated defence response in tobacco. Plant J 18:67-75

Fischer U, Dröge-Laser W (2004) Overexpression of NtERF5, a new member of the tobacco ethylene response transcription factor family enhances resistance to Tobacco mosaic virus. Mol Plant Microbe Interact 17:1162-1171 
Gu YQ, Wildermuth MC, Chakravarthy S, Loh YT, Yang C, He X, Han Y, Martin GB (2002) Tomato transcription factors Pti4, Pti5, and Pti6 activate defense responses when expressed in Arabidopsis. Plant Cell 14:817-831

Hao D, Ohme-Takagi M, Sarai A (1998) Unique mode of GCC box recognition by the DNA-binding domain of ethylene-responsive element-binding factor (ERF domain) in plant. J Biol Chem 273:26857-26861

Hirabayashi S, Matsushita Y, Sato M, Oh-i R, Kasahara M, Abe H, Nyunoya H (2004) Two proton pump interactors identified from a direct phosphorylation screening of a rice cDNA library by using a recombinant BRI1 receptor kinase. Plant Biotechnol 21:35-45

Hiratsu K, Ohta M, Matsui K, Ohme-Takagi M (2002) The SUPERMAN protein is an active repressor whose carboxyterminal repression domain is required for the development of normal flowers. FEBS Lett 514:351-354

Hori K, Watanabe Y (2003) Construction of a tobamovirus vector that can systemically spread and express foreign gene products in solanaceous plants. Plant Biotechnol 20:129-136

Jones JDG, Dangl JL (2006) The plant immune system. Nature 444:323-329

Kagale S, Links MG, Rozwadowski K (2010) Genome-wide analysis of ethylene-responsive element binding factor-associated amphiphilic repression motif-containing transcriptional regulators in Arabidopsis. Plant Physiol 152:1109-1134

Kazan K (2006) Negative regulation of defence and stress genes by EAR motif-containing repressors. Trends Plant Sci 11:109-112

Kim CY, Liu Y, Thorne ET, Yang H, Fukushige H, Gassmann W, Hildebrand D, Sharp RE, Zhang S (2003) Activation of a stressresponsive mitogen-activated protein kinase cascade induces the biosynthesis of ethylene in plants. Plant Cell 15:2707-2718

McGrath KC, Dombrecht B, Manners JM, Schenk PM, Edgar CI, Maclean DJ, Scheible W-R, Udvardi MK, Kazan K (2005) Repressor- and activator-type ethylene response factors functioning in jasmonate signaling and disease resistance identified via a genome-wide screen of Arabidopsis transcription factor gene expression. Plant Physiol 139:949-959

Menke F-LH, Kang H-G, Chen Z, Park JM, Kumar D, Klessig DF (2005) Tobacco transcription factor WRKY1 is phosphorylated by the MAP kinase SIPK and mediates HR-like cell death in tobacco. Mol Plant Microbe Interact 18:1027-1034

Murad L, Lim KY, Christopodulou V, Matyasek R, Lichtenstein CP, Kovarik A, Leitch AR (2002) The origin of tobacco's T genome is traced to a particular lineage within Nicotiana tomentosiformis (Solanaceae). Am J Bot 89:921-928

Nakano T, Suzuki K, Fujimura T, Shinshi H (2006) Genome-wide analysis of the ERF gene family in Arabidopsis and rice. Plant Physiol 140:411-432

Nasir KHB, Takahashi Y, Ito A, Saitoh H, Matsumura H, Kanzaki H, Shimizu T, Ito M, Fujisawa S, Sharma PC, Ohme-Takagi M, Kamoun S, Terauchi R (2005) High-throughput in planta expression screening identifies a class II ethylene-responsive element binding factor-like protein that regulates plant cell death and non-host resistance. Plant J 43:491-505

Nishiuchi T, Suzuki K, Kitajima S, Sato F, Shinshi H (2002) Wounding activates immediate early transcription of genes for ERFs in tobacco plants. Plant Mol Biol 49:473-482

Nishiuchi T, Shinshi H, Suzuki K (2004) Rapid and transient activation of transcription of the ERF3 gene by wounding in tobacco leaves: Possible involvement of NtWRKYs and autorepression. J Biol Chem 279:55355-55361
Ohme-Takagi M, Shinshi H (1995) Ethylene-inducible DNA binding proteins that interact with an ethylene-responsive element. Plant Cell 7:173-182

Ohme-Takagi M, Suzuki K, Shinshi H (2000) Regulation of ethyleneinduced transcription of defense genes. Plant Cell Physiol 41:1187-1192

Ohta M, Ohme-Takagi M, Shinshi H (2000) Three ethyleneresponsive transcription factors in tobacco with distinct transactivation functions. Plant J 22:29-38

Ohta M, Matsui K, Hiratsu K, Shinshi H, Ohme-Takagi M (2001) Repression domains of class II ERF transcriptional repressors share an essential motif for active repression. Plant Cell 13:1959-1968

Park JM, Park C-J, Lee S-B, Ham B-K, Shin R, Paek K-H (2001) Overexpression of the tobacco Tsil gene encoding an EREBP/ AP2-type transcription factor enhances resistance against pathogen attack and osmotic stress in tobacco. Plant Cell 13:1035-1046

Sasaki N, Ogata T, Deguchi M, Nagai S, Tamai A, Meshi T, Kawakami S, Watanabe Y, Matsushita Y, Nyunoya H (2009) Over-expression of putative transcriptional coactivator KELP interferes with Tomato mosaic virus cell-to-cell movement. Mol Plant Pathol 10:161-173

Shinshi H, Usami S, Ohme-Takagi M (1995) Identification of an ethylene-responsive region in the promoter of a tobacco class I chitinase gene. Plant Mol Biol 27:923-932

Soosaar JLM, Burch-Smith TM, Dinesh-Kumar SP (2005) Mechanisms of plant resistance to viruses. Nat Rev Microbiol 3:789-798

Suzuki K, Suzuki N, Ohme-Takagi M, Shinshi H (1998) Immediate early induction of mRNAs for ethylene-responsive transcription factors in tobacco leaf strips after cutting. Plant J 15:657-665

Takabatake R, Ando Y, Seo S, Katou S, Tsuda S, Ohashi Y, Mitsuhara I (2007) MAP kinases function downstream of HSP90 and upstream of mitochondria in TMV resistance gene $N$ mediated hypersensitive cell death. Plant Cell Physiol 48:498-510

Whitham S, Dinesh-Kumar SP, Choi D, Hehl R, Corr C, Baker B (1994) The product of the tobacco mosaic virus resistance gene $N$ : similarity to toll and the interleukin-1 receptor. Cell 78:1101-1115

Yang K-Y, Liu Y, Zhang S (2001) Activation of a mitogen-activated protein kinase pathway is involved in disease resistance in tobacco. Proc Natl Acad Sci USA 98:741-746

Zhang S, Klessig DF (1998) Resistance gene $N$-mediated de novo synthesis and activation of a tobacco mitogen-activated protein kinase by tobacco mosaic virus infection. Proc Natl Acad Sci USA 95:7433-7438

Zhang S, Liu Y (2001) Activation of salicylic acid-induced protein kinase, a mitogen-activated protein kinase, induces multiple defense responses in tobacco. Plant Cell 13:1877-1889

Zhang G, Chen M, Chen X, Xu Z, Guan S, Li L-C, Li A, Guo J, Mao L, Ma Y (2008) Phylogeny, gene structures and expression patterns of the ERF gene family in soybean (Glycine max L.). J Exp Bot 59:4095-4107

Zhang G, Chen M, Li L, Xu Z, Chen X, Guo J, Ma Y (2009) Overexpression of the soybean GmERF3 gene, an AP2/ERF type transcription factor for increased tolerances to salt, drought, and diseases in transgenic tobacco. J Exp Bot 60:3781-3796

Zhuang J, Chen J-M, Yao Q-H, Xiong F, Sun C-C, Zhou X-R, Zhang J, Xiong A-S (2011) Discovery and expression profile analysis of AP2/ERF family genes from Triticum aestivum. Mol Biol Rep $38: 745-753$ 